\title{
FI-Semihollow and FI- Semilifting Modules
}

\author{
Dr.Sahira M. Yaseen ${ }^{1}$, Layla H. Helal ${ }^{2}$ \\ ${ }^{1}$ Department of Math., College of Science, University of Baghdad, Baghdad, Iraq \\ ${ }^{2}$ Department of Civil Eng. College of Eng.University of Baghdad, Baghdad, Iraq
}

\begin{abstract}
Let $R$ be a commutative ring with identity and let $M$ be a left unital Rmodule. In this paper we also give a generalization of semihollow and modules, namely FI- semihollow, FI- semilifting modules respectivly. We study the properties of this concept.
\end{abstract}

Keywords: semihollow module, semilifting module, fully invariant submodule.

\section{Introduction}

Let $\mathrm{R}$ be a commutative ring with identity and $\mathrm{M}$ be a left unital R-module. A submodule $\mathrm{N}$ of $\mathrm{R}$-module $\mathrm{M}$ is called small in $M$ (denoted by $N \ll M$ ) if $N+K \neq M$ for each proper submodule $\mathrm{K}$ of Mand $\mathrm{M}$ is called a hollow module if every proper submodule is small in $\mathrm{M}$, [2]. A submodule $\mathrm{N}$ of R-module $\mathrm{M}$ is called fully invariant submodule of $\mathrm{M}$ if $\mathrm{f}(\mathrm{N}) \subseteq \mathrm{N}$, for every $\mathrm{f} \in \operatorname{Hom}(\mathrm{M}, \mathrm{M})$. Clearly 0and $\mathrm{M}$ are fully invariant submodule of $\mathrm{M}$.The $\mathrm{R}$-module is called duo module if every submodule of $\mathrm{M}$ is fully invariant [3].

In [4] there was given the concepts of semismall submodule semihollow modules and semilifting modules as a generalization of the concepts of small submodule, hollow modules and lifting modules. Where a submodule $\mathrm{N}$ of $\mathrm{R}$ module $\mathrm{M}$ I, is called semismall $\left(\mathrm{N} \ll_{\mathrm{s}} \mathrm{M}\right)$ if $\mathrm{N}=0$ or for each nonzero submodule Kof $M$, then $N / K \ll M / K$.

In this paper we introduce the concept of FI-semihollow module and FI-semilifting module as a generalization of semihollow and semilifting modules and study the basic properties of this type of modules and give some characterizations for such modules.

\section{Lemma (1):[4]}

Let $\mathrm{N}$ be a submodule of R-module $\mathrm{M}, \mathrm{N} \ll_{\mathrm{s}} \mathrm{M}$ iff $\mathrm{N}+\mathrm{L}=\mathrm{M}$ for all $\mathrm{L} \leq \mathrm{M}$ implies $\mathrm{K}+\mathrm{L}=\mathrm{M}$ For all $\mathrm{K} \leq \mathrm{N}, \mathrm{K} \neq(0)$.

Lemma (2):[4]

1) IF $\mathrm{N} \ll_{\mathrm{s}} \mathrm{M}$ and $\mathrm{A}<\mathrm{N}$ then $\mathrm{A} \ll_{\mathrm{s}} \mathrm{M}$.

2) IF $X, Y$ are submodule of $M$ such that $X \ll_{s} Y$ then $X \ll_{s}$ $\mathrm{M}$.

3) IF $\mathrm{N} \ll_{\mathrm{s}} \mathrm{M}$ and $\mathrm{K}<\mathrm{M}$ such that $\mathrm{K} \subseteq \mathrm{N}$ then $\mathrm{N} / \mathrm{K} \ll_{\mathrm{s}} \mathrm{M} / \mathrm{K}$.

4) Let $\mathrm{M}=\mathrm{M}_{1} \oplus \mathrm{M}_{2}$ and $\mathrm{N}<\mathrm{M}$ such that $\mathrm{N}=\mathrm{N}_{1} \oplus \mathrm{N}_{2}$, IF $\mathrm{N} \ll_{\mathrm{s}}$ $M$ then $\mathrm{N}_{1} \ll_{\mathrm{s}} \mathrm{M}_{1}$ and $\mathrm{N}_{2} \ll_{\mathrm{s}} \mathrm{M}_{2}$

5) Let $\mathrm{N}<\mathrm{N}_{1}<\mathrm{M}$ if $\mathrm{N} \ll_{\mathrm{s}} \mathrm{M}$ and $\mathrm{N}_{1}$ is direct summand then $\mathrm{N} \ll \mathrm{s}_{\mathrm{s}}$.

\section{1- FI-Semi Hollow module}

An non-zero R-module $M$ is called semihollow iff every proper submodule of $\mathrm{M}$ is Semismall, [4].In this section we introduce the concept of FI-semihollow module and we study the properties of this concept.
Definition (1.1):-Anon zero R-module $M$ is called FIsemihollow if every fully invariant proper submodule of $\mathrm{M}$ is Semismall.

A ring $\mathrm{R}$ is called FI-semihollow if $\mathrm{R}$ is FI-semihollow as an $\mathrm{R}$-module , Equivalently every two sided ideal of $\mathrm{R}$ is semismall.

Remarks and Examples (1.2):-

1) Every hollow and semihollow is FI-semihollow module.

2) IF M is Duo module then semihollow and FI-semihollow are equivelent.

3) $\mathrm{Z}$ as Z-module is not semihollow and not FI-semihollow since $2 Z$ is fully invariant proper submodule and $2 Z+3 Z=Z$ but $6 Z+3 Z \neq M, 6 Z<2 Z$

4) $\mathrm{Z}_{2} \oplus \mathrm{Z}_{8}$ as $\mathrm{Z}$-module is FI-semihollow which is not Duo module.

5) Every simple R-module is FI-semihollow

\section{Proposition (1.3):}

FI-semihollow is closed under isomorphism.

\section{Proof:}

Let $\mathrm{M}, \mathrm{M}^{\prime}$ be R-modules and $\mathrm{M}$ is FI-semihollow, let $\mathrm{f}$ : $\mathrm{M} \rightarrow \mathrm{M}^{\prime}$ be an R-isomorphism, we have to show that $\mathrm{M}^{\prime}$ is FIsemihollow, let $\mathrm{N}$ be fully invariant proper Submodule of $\mathrm{M}^{\prime}$, Now, $\mathrm{f}^{-1}(\mathrm{~N})$ is proper submodule of $\mathrm{M}$, If $\mathrm{f}^{-1}(\mathrm{~N})=\mathrm{M}$ then $\mathrm{f}\left(\mathrm{f}^{-1}(\mathrm{~N})\right)=\mathrm{N}=\mathrm{M}^{\prime}$ which is comtoduction. Thus $\mathrm{f}^{-1}(\mathrm{~N})$ is proper submodule if $\mathrm{M}$ to show $\mathrm{f}^{-1}(\mathrm{~N})$ is fully invariant of M.Let $\mathrm{g}$ : $\mathrm{M} \rightarrow \mathrm{M}$, since $\mathrm{N}$ is fully invariant of $\mathrm{M}^{\prime}$, thus $\mathrm{fgf}^{-1}(\mathrm{~N}) \subseteq \mathrm{N}$ and $\mathrm{f}^{-1} \mathrm{f} \quad\left(\mathrm{g}\left(\mathrm{f}^{-1}(\mathrm{~N})\right) \subseteq \mathrm{f}^{-1}(\mathrm{~N})\right.$, then $\mathrm{g}\left(\mathrm{f}^{-1}(\mathrm{~N})\right) \subseteq \mathrm{f}^{-1}(\mathrm{~N})$. Thus $\mathrm{f}^{-1}(\mathrm{~N})$ is fully invariant of $\mathrm{M}$, since $\mathrm{M}$ is FI-semihollow , thus $\mathrm{f}^{-1}(\mathrm{~N}) \ll_{\mathrm{s}} \mathrm{M}$ and by [4] prop. (1.3) and $\left(\mathrm{N} \ll_{\mathrm{s}} \mathrm{M}^{\prime}\right)$.

\section{Proposition (1.4):}

Let $\mathrm{M}$ be a FI- semihollow and $\mathrm{N}$ be a submodule of $\mathrm{M}$ with $\mathrm{N} / \mathrm{K}$ is direct summand of $\mathrm{M} / \mathrm{K}$ for each proper submodule $\mathrm{K}$ of $\mathrm{N}$ then $\mathrm{N}$ is FI-semihollow.

Proof: Let L be a proper fully invariant submodule of $\mathrm{N}$ then $\mathrm{L} \ll_{\mathrm{s}} \mathrm{M}$, by $\operatorname{Lemma}(2)$.

Let $\mathrm{K}$ be a proper submodule of $\mathrm{L}$ then $\mathrm{L} / \mathrm{K} \ll \mathrm{M} / \mathrm{K}$ and by hypothesis $\mathrm{L} / \mathrm{K}$ is a direct summund of $\mathrm{M} / \mathrm{K}$ then $\mathrm{L} / \mathrm{K} \ll_{\mathrm{s}}$ $\mathrm{N} / \mathrm{K}[4]$, hence $\mathrm{L} \ll{ }_{\mathrm{s}} \mathrm{N}$ 


\section{International Journal of Science and Research (IJSR) \\ ISSN (Online): 2319-7064}

Index Copernicus Value (2015): 78.96 | Impact Factor (2015): 6.391

Remark ( 1.5): FI-semihollow need not be indecomposable , $\mathrm{Z}_{6}$ as Z-module is semihollow then FI-semihollow which is decomposable.

Recall that a submodule $\mathrm{N}$ of an $\mathrm{R}$-module $\mathrm{M}$ is called coclosed in $M$ if whenever $N / K^{\prime} \ll M / K$ then $N=K \forall K$ submodule of $\mathrm{M}$ contained in $\mathrm{N}[5]$

This means $\mathrm{N}$ is coclosed if whenever $\mathrm{K}<\mathrm{N}, \mathrm{N} / \mathrm{K}$ is not small in $\mathrm{M} / \mathrm{K}$, it is known that the only proper coclosed submodule in semihollow module is simple submodule.

We have the following Remark.

Remark: Every proper fully invariant coclosed submodule of a semihollow module is a simple submodule.

Proof: let $\mathrm{N}$ be fully proper coclosed submodule of FIsemihollow module $M$, since $M$ is FI-semihollow then $N / K$ $\ll \mathrm{M} / \mathrm{K} \forall \mathrm{K} \leq \mathrm{N}$ since $\mathrm{N}$ is coclosed , $\mathrm{N}=\mathrm{K}$ then $\mathrm{N}$ issimple.

Corollary: Every non-zero coclosed fully invariant submodule of FI-semihollow module is FI-semihollow.

Proof: Since every simple is FI-semihollow.

\section{FI-Semilifting modules}

An R-module $\mathrm{M}$ is called semilifting if for any submodule $\mathrm{N}$ of $M$ there exist submodules $K, K^{\prime}$ of $M$ such that $M=K \oplus K^{\prime}$ with $\mathrm{K} \leq \mathrm{K}$ and $\mathrm{N} \cap \mathrm{K}^{\prime} \ll_{\mathrm{s}} \mathrm{N}$ (equivalently $\mathrm{N} \cap \mathrm{K}^{\prime} \ll_{\mathrm{s}} M$ ). In this section we introduce the notion of FI-semilifting modules and discus some properties of this kind of modules which are generalization of semilifting module.

\section{Definition (2.1) :}

An R-module $\mathrm{M}$ is called FI-semilifting if for any fully invariant submodule $\mathrm{N}$ of $\mathrm{M}$ there exist submodules $\mathrm{K}, \mathrm{K}^{\prime}$ of $M$ such that $M=K \oplus K^{\prime}$ with $K \subseteq N$ and $N \cap K^{\prime} \ll_{s} N$. [4] The following theorem given a characterization of FI-semilifting modules.

Theorem (2.2) :Let $\mathrm{M}$ be an R-module,then the following statement are equivalent :

1) $\mathrm{M}$ is FI-semilifting

2) Every fully invariant submodule $N$ of $M, N$ can be written as $\mathrm{N}=\mathrm{A} \oplus \mathrm{B}$ where $\mathrm{A}$ is direct summand of $\mathrm{M}$ and $\mathrm{B} \ll_{\mathrm{s}} \mathrm{M}$.

3) For every fully invariant submodule $\mathrm{N}$ of $\mathrm{M}$, there exist a direct summand $K$ of $M$ such that $K \leq N$ and $N / K \ll_{s}$ $\mathrm{M} / \mathrm{K}$.

Proof:It is clearsame like theorem 3.3 in [4].

It is known that every hollow is lifting, but the converse is not true (see Remark 1.1.7. in 11).

Remark (2.3) :

1) Every FI-semihollow is FI-semilifting

Proof: - let $\mathrm{N}$ be fully invariant submodule of $\mathrm{M}$ if $\mathrm{N} \neq \mathrm{M}$, $\mathrm{N} \ll_{\mathrm{s}} \quad \mathrm{M}, \mathrm{N}=(0) \oplus \mathrm{N}$ the result follows directly by (theorem2.2)

2) Every semisimple module is lifting hence semilifting and then FI- semilifting.
Proposition (2.4) :

An indecomposable R-module is FI-semihollow if and only if FI-semilifting.

Proof: Let M be FI-semihollow then M is FI-semilifting by Remark (2.3)

Conversely suppose that $\mathrm{M}$ is FI-semilifting and A fully invariant proper submodule of $\mathrm{M}$, by (Theorem.2.2), We have $A=N \oplus D$ where $N$ is a direct summand of $M$ and $D \ll$ $M$ but $\mathrm{M}$ is indecomposable. Then either $\mathrm{N}=(0)$ or $\mathrm{N}=\mathrm{M}$ then $\mathrm{M}=\mathrm{N} \subseteq \mathrm{A}$ which implies that $\mathrm{A}=\mathrm{M}$ which is contradiction, thus $\mathrm{N}=(0)$, So $\mathrm{A}=\mathrm{D} \ll_{\mathrm{s}} \mathrm{M}$, hence $\mathrm{M}$ is FIsemihollow.

\section{Proposition (2.6) :}

If an R-module $\mathrm{M}$ is FI- semihollow then $\mathrm{M} / \mathrm{N}$ is FIsemihollow for every fully invariant proper submodule $\mathrm{N}$ of M.

Proof: Assume that $\mathrm{M}$ is FI-semihollow and let $\mathrm{N}$ be fully invariant proper submodule of $\mathrm{M}$

Let $\mathrm{K} / \mathrm{N}$ be fully invariant proper submodule of $\mathrm{M} / \mathrm{N}$,Such that $\frac{\mathrm{M}}{\mathrm{N}}=\frac{\mathrm{K}}{\mathrm{N}}+\frac{\mathrm{H}}{\mathrm{N}}$ where $\mathrm{H} \subseteq \mathrm{M}$ and $\mathrm{N} \subset \mathrm{H}$. Then $\frac{\mathrm{M}}{N}=\frac{K+H}{N}$, So it implies $M=K+H$, now since $K / N$ is fully invariant of $M / N$ and $\mathrm{N}$ is fully invariant proper of $\mathrm{M}$ thus $\mathrm{K}$ is fully invariant by (lemma 1.2.23[6]) then $\mathrm{K} \ll_{\mathrm{s}} \mathrm{M}$, So $\mathrm{K}+\mathrm{H}=\mathrm{M}, \mathrm{H} \leq \mathrm{M}$ then $\mathrm{K}^{\prime}+\mathrm{H}=\mathrm{M}, \forall \mathrm{K}^{\prime} \subset \mathrm{K}\left(\right.$ Lemma1). Hence we have $\frac{\mathrm{K}^{\prime}+H}{N}=\frac{\mathrm{M}}{\mathrm{N}}$ then $\frac{M}{N}=\frac{\mathrm{K}^{\prime}}{N}+\frac{H}{N} \forall \frac{\mathrm{K}^{\prime}}{N} \subseteq \frac{K}{N}, \mathrm{~K}^{\prime} \neq \mathrm{N}$, thus $\frac{M}{N}$ is FI- semihollow module.

\section{References}

[1] D.Kasch,Modules and rings ,Acadimic Press,London, 1982

[2] P.Feury, "Hollow Modules and Local Endomorphism Rings", pac.J.Math., 53 (1974), 379-385.

[3] A.C. Ozcan,Duo Modules, Glasgow math.J.Trust 48,2006,pp.533-545.

[4] I.M.Ali and L.S.Mahmood, "Semismall submodules and Semi-lifting Modules", Proc. of $3^{\text {rd }}$ Scientific Conference of College of Science, University of Baghdad 24-26 March(2009), 385-393.

[5] Y.Talebi and T.amoozegar fully invariant TM- Lifting Module,AlbanianJ.of Math. V.3(2009), 49-53,ISSN 1930-1235.

[6] Y. Talebi and T.AmoozeGar, strong FI-Lifting module.International Electronic J.of Algebra 3(2008), 75-82. 